\title{
EL DESARROLLO PROFESIONAL DEL INDIVIDUO EN LAS TEORÍAS DE LA ORGANIZACIÓN: UNA MIRADA CRÍTICA
}

\author{
THE PROFESSIONAL DEVELOPMENT OF THE INDIVIDUAL WITHIN ORGANIZATIONAL \\ THEORIES: A CRITICAL PERSPECTIVE
}

\author{
Cristina Robledo-Ardila ${ }^{a}$ \\ Clasificación: trabajo conceptual \\ Recibido: 16-julio-2020 / Revisado: 28-septiembre-2020 / Aceptado: 1-octubre-2020
}

\section{Resumen}

Este artículo hace un análisis conceptual acerca de cuál es el lugar que ocupa el desarrollo profesional del individuo dentro de la Teoría de la Organización (TO) como un asunto organizacional. Para ello, en la primera parte se explican los elementos generales de la TO respecto a dicho tema. En un segundo momento, se analizan los elementos que diferentes escuelas proponen para el estudio del desarrollo profesional del individuo en la organización. Como resultado de esa lectura, se concluye que 1) si bien el individuo está presente con bastante frecuencia en las TO, lo hace principalmente a través de las interacciones sociales en la organización, y 2) la consideración de sus necesidades y objetivos individuales se da en relación con los de la organización. Ambos aspectos revelan una racionalidad altamente instrumental de la TO respecto al desarrollo profesional del individuo.

Palabras clave: Teoría de la Organización, individuo, desarrollo

\begin{abstract}
This paper offers a conceptual analysis of the postulates of different schools of thought within the Organizational Theories in order to comprehend the role played by the process of professional development of the individual within this field of study, and as an organizational issue. For this purpose, the first section outlines the key elements referring to the role of individuals in organizational theory; then, a critical revision and interpretation of the main postulates of diverse schools are presented. As a result, it was found that the individual is present through the different schools but very often the focus is on the social relations that take place in the organization, and the organizational goals as the common goals that all members must pursue, as opposed to his individual goals and needs. This way, organizational theories display an instrumental rationality towards the topic of this paper.
\end{abstract}

Keywords:Organizational theory, individual, development

a Universidad EAFIT, Departamento de Negocios Internacionales. Medellín, Colombia. Correo electrónico: crobled1@eafit.edu.co 


\section{Introducción}

De acuerdo con los planteamientos de Scott (1981), el estudio de las organizaciones es un campo especializado derivado de la sociología con un enfoque multidisciplinario. Si bien es una disciplina imperfecta, ha sido "una de las preocupaciones fundamentales de los investigadores en un mundo industrializado" (Ibarra-Colado y Montaño, 1991, p. 7), en cuanto busca constituirse como una teoría de lo organizado. La importancia del estudio de las organizaciones estriba en que estas se constituyen en "un componente dominante de la sociedad contemporánea" (Hall, 1996a, p. 1), son la base para "las grandes transformaciones sociales en la historia" (Hall, 1996a, p. 2) y su estudio permite, como lo plantea Perrow (1980), "sobrevivir entre y dentro de ellas" (p. 307). Valga aclarar que no todo es una organización (Scott, 1981) ni se puede clasificar como organizacional (Hall, 1996a), pero sí puede afirmarse que las organizaciones generan resultados para los individuos, para la comunidad y para la sociedad, de forma tal que se constituyen como una extensión del individuo, pues le permiten perseguir y alcanzar metas que están fuera de su alcance particular (Scott, 1981).

En lo que se refiere al individuo en la organización, en los planteamientos generales de la TO se encuentran tres elementos fundamentales que justifican la exploración de este campo en la búsqueda de marcos teóricos útiles para el estudio de los procesos de desarrollo profesional del individuo en la organización: la presencia de la organización en todos los ámbitos de su existencia y el individuo como participante de la organización, el individuo como nivel de análisis de la TO y el individuo como receptor de los resultados de la organización.

Al revisar las definiciones de las organizaciones que ofrece la TO, se encuentra que existen numerosas perspectivas y definiciones bajo las cuales resulta interesante analizar el desarrollo del individuo en la organización. Hall (1996a) resalta las contribuciones de Weber (1922), Barnard (1959) y algunas perspectivas marxistas en la búsqueda de una definición de organización. En ese orden de ideas, Weber (1922) reconoce la existencia de las relaciones sociales, así como la frontera que separa a la organización y a sus miembros de otras organizaciones; entre tanto, Barnard enfatiza en los individuos y la coordinación que debe existir entre ellos para lograr un propósito común, $\mathrm{y}$, finalmente, Marx aporta la noción de la praxis o la actividad práctica humana, tanto individual como colectiva, con el fin de conseguir un resultado. A partir de estos planteamientos, Hall (1996b) propone definir una organización como

Una colectividad con una frontera relativamente identificable, un orden normativo, niveles de autoridad, sistemas de comunicaciones y sistemas de coordinación de membresías; esta colectividad existe de manera continua en un ambiente y se involucra en actividades que se relacionan por lo general con un conjunto de metas; las actividades tienen resultados para los miembros de la organización, la organización misma y la sociedad. (p. 33)

Esta definición reconoce la existencia del individuo de tres maneras importantes: como miembro de la organización, como ser social y como contribuyente para alcanzar el propósito de la organización como un objetivo común.

Ahora bien, Scott (1981) identifica tres niveles de análisis de la organización: (1) el comportamiento o atributos de los individuos en la organización, donde la psicología social ocupa un lugar importante y donde sobresalen las obras de autores como March y Simon (1969), Porter, Lawler y Hackman (1975); (2) el funcionamiento o características de algún aspecto o segmento de la estructura, también conocido como el nivel estructural que se enfoca en las subdivisiones de la organización, así como en las interrelaciones entre ellas; y (3) las características o acciones de la organización conocidas como el nivel ecológico que asume la organización como un ente colectivo que funciona dentro de un entorno complejo caracterizado por un gran número de organizaciones interdependientes. Entonces, el individuo aparece como un elemento en el estudio de la TO cuando se considera como uno de los niveles de análisis y se reconoce el papel que desempeñan las diferencias individuales dentro de esta disciplina.

Hall (1996b) plantea tres niveles de receptores de los resultados de la organización, cada uno de ellos conformado por individuos, así: los individuos mismos, las comunidades y la sociedad. En este sentido, podría decirse que el individuo es un actor clave, bien sea como participante o como receptor, de todos los resultados generados por la organización, y, en consecuencia, su desarrollo debe ser un tema organizacional. Adicionalmente, si se considera a la organización como el único mecanismo mediante el cual se pueden lograr metas y objetivos (Parsons, 1960, citado en Scott, 1981), el tema cobra aún más relevancia.

El individuo como receptor de los resultados de la organización es influenciado mediante diversos factores como la división del trabajo, las condiciones y los cambios demográficos organizacionales, e incluso la estratificación social como consecuencia del quehacer organizacional y como medio para el progreso de los individuos (Hall, 1996a). Pero los efectos que recibe el individuo de la organización no se limitan a su rol de trabajador como participante de esta. El individuo, en cuanto miembro de la sociedad, también se ve afectado por los resultados 
de esta, especialmente si se tiene en cuenta que en algunos casos las organizaciones sirven a los intereses de ciertos individuos o grupos para permitir que "una clase reinante mantenga su poder y riqueza a costa del resto de la población" (Hall, 1996a, p. 11).

La organización tiene una existencia legal equiparable con la de una persona natural, lo cual no es más que el resultado de la acción conjunta de los individuos que la conforman, a quienes la organización influencia a través de la alteración de los patrones de trabajo y los cambios en su distribución, generando cambios en ellos que se extienden a las demás esferas de su vida (Hall, 1996b). Esto implica que las organizaciones son reales para los individuos "en el grado en que los factores estrictamente organizacionales son responsables por parte del comportamiento de los individuos en todo tiempo dentro de las organizaciones" (Hall, 1996b, p. 35) y, por ende, perduran en el tiempo y trascienden a sus miembros.

Estos planteamientos pueden considerarse como una invitación a profundizar en los procesos individuales a través de la TO y a asumir como categorías del análisis conceptual propuesto aquí al individuo como participante y receptor, y como unidad de análisis en el estudio de los temas organizacionales. Para este fin, a continuación, se presenta una revisión de algunas de las escuelas de la TO, cuya selección obedece a criterios de pertinencia y relevancia con respecto al problema central de la investigación en la que se enmarca este artículo, a saber, el lugar que ocupa el desarrollo profesional del individuo dentro de la TO como un asunto organizacional, el individuo y las escuelas de la TO.
Como lo sugiere Perrow (1980), existe un gran número de teorías que ofrecen respuesta a diversidad de intereses en torno al estudio de las organizaciones, de manera que se hace necesario que el investigador seleccione entre ellas a aquellas afines a sus intereses temáticos y propósitos investigativos. Para este caso, los intereses y propósitos pueden enunciarse a partir de la siguiente pregunta: ¿el papel de individuo en las organizaciones puede constituirse en un factor que determina el desarrollo profesional del mismo?. En este sentido, cabe resaltar que las escuelas presentadas a continuación permiten estudiar la evolución conceptual de la noción de individuo en el contexto de las organizaciones y su papel en ellas desde una perspectiva histórica. Es importante mencionar que la lista no tiene una vocación exhaustiva y se eligen aquellas representativas de cambios conceptuales determinantes. Su selección obedece a su función epistemológica como seminales de la organización, toda vez que a diferencia de las teorías de la administración (TA) no tienen un carácter prescriptivo ni se enfocan en la gestión (Gonzales-Miranda y Gentilin, 2012), sino que su objeto es describir a las organizaciones en términos de sus componentes, sus formas, sus configuraciones y sus elementos estructurales. La tabla 1 presenta un resumen de los resultados de esta revisión a la luz de la pregunta de investigación planteada.

\section{La base material como antecedente de la TO}

Si bien esta escuela no hace parte de la TO, es importante como antecedente de propuestas posteriores, ya que

Tabla 1.Visión del individuo en las teorías de la organización

\begin{tabular}{|c|c|c|c|}
\hline Teoría & Supuestos & Preocupación & Limitaciones \\
\hline $\begin{array}{l}\text { La base material como } \\
\text { antecedente de la TO }\end{array}$ & $\begin{array}{l}\text { El trabajador es mentiroso, los sistemas } \\
\text { de administración son defectuosos. }\end{array}$ & $\begin{array}{l}\text { Aumentar la producción por unidad } \\
\text { de esfuerzo humano. }\end{array}$ & $\begin{array}{l}\text { Perspectiva altamente funcionalista con respecto } \\
\text { al papel del individuo en la organización. }\end{array}$ \\
\hline $\begin{array}{l}\text { Escuela de las Relaciones } \\
\text { Humanas }\end{array}$ & $\begin{array}{l}\text { Las diferencias individuales pueden } \\
\text { alterar el equilibrio y afectar su } \\
\text { rendimiento en el trabajo. }\end{array}$ & $\begin{array}{l}\text { Aprender nuevos métodos para } \\
\text { comprender a los hombres y para } \\
\text { encauzar situaciones perfectamente } \\
\text { humanas. }\end{array}$ & $\begin{array}{l}\text { Racionaliza el factor humano como actor en las } \\
\text { relaciones sociales que se dan al interior de la } \\
\text { organización, no como individuo. }\end{array}$ \\
\hline Escuela de la Burocracia & $\begin{array}{l}\text { El individuo como trabajador y } \\
\text { funcionario profesional que no puede } \\
\text { desprenderse de la organización. }\end{array}$ & $\begin{array}{l}\text { La profesionalización del individuo } \\
\text { y la posibilidad de progresar en la } \\
\text { organización. }\end{array}$ & $\begin{array}{l}\text { Objetividad racional mediante la educación y la } \\
\text { meritocracia. }\end{array}$ \\
\hline Escuela del Comportamiento & $\begin{array}{l}\text { El individuo contribuye a las metas de } \\
\text { la organización a cambio de un salario. }\end{array}$ & $\begin{array}{l}\text { Despojar al individuo de su autonomía } \\
\text { y sustituirla por un proceso organizado } \\
\text { para la toma de las decisiones. }\end{array}$ & $\begin{array}{l}\text { Perspectiva determinista que ignora a los } \\
\text { individuos como sujetos con metas e intereses } \\
\text { propios. }\end{array}$ \\
\hline Escuela de la Contingencia & $\begin{array}{l}\text { Diferentes condiciones externas pueden } \\
\text { necesitar características organizativas } \\
\text { diferentes. }\end{array}$ & $\begin{array}{l}\text { La organización informal como un } \\
\text { sistema de relaciones sociales de } \\
\text { interdependencia y sus efectos sobre } \\
\text { la organización. }\end{array}$ & $\begin{array}{l}\text { No reconoce la complejidad del individuo } \\
\text { como parte fundamental de la complejidad de la } \\
\text { organización. }\end{array}$ \\
\hline $\begin{array}{l}\text { Escuela de las Nuevas } \\
\text { Relaciones Humanas }\end{array}$ & $\begin{array}{l}\text { La organización es racional y hace } \\
\text { demandas racionales a sus empleados. }\end{array}$ & $\begin{array}{l}\text { La participación del individuo en la } \\
\text { organización se consigue mediante } \\
\text { la motivación. }\end{array}$ & $\begin{array}{l}\text { Racionalidad instrumental y funcionalismo: } \\
\text { reconoce la subjetividad del individuo solo en } \\
\text { función del propósito de la organización. }\end{array}$ \\
\hline
\end{tabular}


algunos de sus planteamientos iluminan su comprensión. La obra de Taylor marcó "el rumbo de la organización del trabajo en las organizaciones modernas por un siglo" (Barba Álvarez, 2010, p. 17) mediante la búsqueda de la eficiencia. Desde los orígenes mismos de la administración científica, Taylor (1997) hace un llamado hacia un cambio de mentalidad tanto en los trabajadores como en los patrones, con el fin de lograr el trabajo conjunto para incrementar el superávit para todos, en lugar de enfrentarse por su división; es decir, la sustitución del antagonismo por la cooperación, que en adelante será la base de planteamientos posteriores. Considera Taylor (1997) que el trabajador es mentiroso y que los sistemas de administración son defectuosos de manera que el haraganeo y el bajo rendimiento sistemático son preocupaciones fundamentales de esta escuela que tiene como su principal foco el aumentar la producción por unidad de esfuerzo humano.

Podría decirse que el individuo aparece en esta escuela como el propietario del conocimiento, que es su capital más preciado y que habrá que despojarlo de este con el fin de liberar el trabajo para el desarrollo del capital (Coriat, 2000). Solo así surgirá una ciencia que sustituya el conocimiento empírico mediante la aplicación de los principios de la administración científica (Taylor, 1997). Como resultado de esto aparece la separación del trabajo y el conocimiento a través del trabajo mecánico y el trabajo científico, la reducción del conocimiento empírico a fórmulas y leyes, así como la labor y la bonificación como elementos fundamentales del mecanismo de la administración científica. En este sentido, se sugiere que el individuo trabaja a cambio de una bonificación o recompensa que solo aumenta en función de un mayor rendimiento. De la misma forma, se plantea que la estandarización de los procesos en la búsqueda del one best way permite al trabajador realizar un trabajo mejor y más interesante, sin que esto se constituya como una degradación del trabajador (Taylor, 1997). No obstante, es interesante señalar que, al liberarlo de toda responsabilidad de pensar el trabajo y reducir su labor a la colaboración para lograr un mayor rendimiento, se adopta desde ese momento una perspectiva funcionalista con respecto al papel del individuo en la organización. Con la desaparición del obrero de oficio no hay cabida para pensar en que éste se pueda desarrollar como profesional al interior de la organización. El individuo se considera entonces como un robot, lo cual perjudica la calidad de su trabajo y su moral, así como el aprendizaje por limitar el trabajo a tareas simples y repetitivas al punto que puede generar una "atrofia cerebral" (Durand, 1979, p. 64).

$\mathrm{Al}$ respecto dice Coriat (2000), que la administración científica "ha trastocado el antiguo orden de las cosas y de los hombres, que el cronómetro apareció como instrumento de dominación para transformar la producción en un código formal generando un ataque contra el obrero profesional y conllevará más adelante a una crisis del trabajo industrial" (p. 1). En este sentido, Durand (1979) dice:

El taylorismo no solamente ha quebrado las relaciones humanas en la empresa; ha ampliado la separación entre actividades manuales reducidas a tareas de ejecución y actividades intelectuales. El obrero ha sido desapropiado del conocimiento racional de la materia, del producto y de la técnica convertida en el patrimonio de especialistas. (p. 67)

Como consecuencia de esto, se empieza a tomar conciencia del uso de los recursos humanos para conseguir el balance económico de la organización.

\section{La Escuela de las Relaciones Humanas}

Ante un contexto caracterizado por el fracaso corporativo en Estados Unidos y la necesidad de mejorar el estado de ánimo de los trabajadores para su colaboración eficaz (Mayo, 1972), el desgaste del taylorismo, como lo sugiere Durand (1979), llevará al reconocimiento del factor humano y de los factores psicológicos y sociales y a un cambio de actitud frente a la industria, para prestar mayor atención a la vida social en la organización. El fracaso del experimento Hawthorne motivó la indagación hacia nuevos factores que influencian el trabajo, entre ellos aparecen una mayor libertad, mejor actitud mental y mayor goce en el trabajo (Mayo, 1972). Estos resultados dan origen a nuevos estudios con el fin de "aprender nuevos métodos para comprender a los hombres y para encauzar situaciones perfectamente humanas" (Mayo, 1972, p. 95) y reconocer que las diferencias individuales pueden alterar el equilibrio y afectar el rendimiento en el trabajo.

Mayo (1977) llama la atención sobre el desprecio por lo humano y lo social en las organizaciones, debido a que esta situación genera una ruptura social donde se incrementa el número de individuos infelices y hostiles por la destrucción de las relaciones histórico-sociales y personales. En este sentido, el autor sugiere que los individuos en las organizaciones requieren de habilidades técnicas que se relacionan con la capacidad de manipular cosas al servicio de los propósitos humanos, y habilidades sociales que se relacionan con la capacidad para recibir comunicaciones y responder a las actitudes e ideas de los otros. El equilibrio será entonces el resultado del balance entre estos dos tipos de habilidades, lo cual es para Mayo (1977) la causa de los desequilibrios generados previos a esta escuela. 
Si bien bajo esta escuela se reconoce el factor humano como fundamental en la organización, siguiendo los planteamientos de Roesthlisberger y Dickson (1966), quienes sugieren que la organización es un sistema social que debe ser considerado como un todo donde cada parte tiene una relación de interdependencia con las demás, podría decirse que es precisamente este el énfasis de esta escuela: el factor humano como actor en las relaciones sociales dadas al interior de la organización y no el individuo como nivel de análisis en sí mismo. Dichas relaciones sociales son una de las principales fuentes de desequilibrio en cuanto ellas determinan la capacidad de los grupos para trabajar juntos y satisfacer sus propios deseos (Roethlisberger y Dickson, 1966). En ese orden de ideas, es posible decir que los planteamientos de esta escuela someten al individuo a la existencia y preponderancia de un objetivo común, el de la organización, y dejan de lado sus objetivos personales que tan solo se enuncian, pero no se desarrollan.

De acuerdo con Roesthlisberger y Dickson (1966), esta escuela reconoce la existencia de una organización formal y una informal, lo cual es un elemento clave para el análisis del desarrollo del individuo en la organización. Sin embargo, al considerar a la organización informal, el foco está en las relaciones sociales que ocurren informalmente en la organización, más que en los procesos individuales. Asimismo, en esta escuela se plantea la existencia de una organización humana donde cada miembro trae consigo experiencias sociales y personales, en las que se reconoce la pluralidad de los individuos y, especialmente, las relaciones entre ellos. Para los autores, la colaboración humana está determinada por más que la organización formal y la informal. Ese es precisamente el foco de esta escuela: la organización como sistema social estará en equilibrio en cuanto esté integrada por "individuos cuyos sentimientos e intereses son mutuamente dependientes" (Desmarez, 1986, p. 29). Dentro de esta perspectiva aparece el individuo como fuente de desequilibrio de la organización, que es un sistema socio-técnico, pero no hay lugar para el estudio del individuo y su desarrollo en ella, sino siempre en relación con los demás miembros de la organización para alcanzar un objetivo común.

En resumen, la Escuela de las Relaciones Humanas reconoce la existencia del factor humano, pero mantiene como foco la productividad y no devuelve al individuo lo que la Administración Científica le quitó, simplemente lo racionaliza con el objetivo de lograr, ante la presencia de un contexto difícil, su colaboración con el propósito de la organización. De esta forma, no hay aún en esta escuela un lugar para el estudio de las diferencias individuales y su influencia sobre los temas organizacionales. Se reconoce el papel que cumple el individuo en el equilibrio organizacional y se reconoce incluso como fuente de desequilibrio, sin concebirse el equilibrio individual como una forma de mantener la estabilidad de la organización. Podría decirse también que al estudiar los elementos que influencian la colaboración del individuo en la organización se sientan las bases para luego considerar al individuo un activo más.

\section{La Escuela de la Burocracia}

De acuerdo con Weber (1922), la dominación es la única forma de "convertir una acción comunitaria amorfa en una asociación racional [y] es uno de los más importantes elementos de la acción comunitaria. En rigor, no toda acción comunitaria ofrece una estructura de este tipo" (p. 695). Según el autor, la dominación es posible mediante los intereses, es decir, la influencia, o mediante la autoridad, en otros términos, el poder de mando y el deber de la obediencia. De acuerdo con el autor, la dominación legalracional aparece como la dominación mediante las reglas en sí mismas, con independencia de las personas y reduciendo al máximo sus motivos o sentimientos personales con el fin de liberarse de la arbitrariedad y el capricho.

Bajo esta escuela el cargo se convierte en profesión y se configura gracias a la estimación social, el nombramiento por parte de una autoridad superior y que ese cargo se ejerza a perpetuidad. El funcionario recibirá un salario fijo, pues se paga por las funciones y no por la cantidad de trabajo, acompañada de una pensión al momento de su retiro por vejez (Weber, 1922). En este contexto, aparece el funcionario profesional como aquel que no puede desprenderse de la organización y al que se "encargan cometidos especializados dentro de un mecanismo en marcha incesante que únicamente puede ser movido o determinado por la autoridad superior y que es la que le prescribe la ruta determinada" (Weber, 1922, p. 741). Se observa entonces la profesionalización del individuo como trabajador y aparece también la posibilidad de progresar en la organización, es decir, de desarrollo profesional para el individuo basado en la objetividad racional mediante la educación y la meritocracia.

De acuerdo con Mouzelis (1967), el tipo ideal de la burocracia como una conceptualización a partir de elementos empíricos tiene unas características que se evidencian, en mayor o menor medida, en los rasgos de las organizaciones e incluyen el alto grado de especialización, la estructura jerárquica de autoridad con áreas bien definidas en términos de competencia y responsabilidad, el reclutamiento de personal con base en su capacidad y conocimiento técnico, y una clara diferenciación entre la renta y la fortuna privadas. Si bien este tipo en su "pureza ideal no se encuentra nunca en la realidad" 
(Mouzelis, 1967, p. 45), su planteamiento evidencia una serie de reglas racionales que buscan controlar la organización con base en el conocimiento. Esto implica, según el autor, que la libertad individual se vea disminuida mediante la reducción de la discrecionalidad por la existencia de reglas estrictas que no dejan lugar a la iniciativa personal (Mouzelis, 1967). En consecuencia, la Escuela de la Burocracia puede considerarse como altamente formulista, pues supone una distribución de funciones, un sistema de relaciones prescritas y una serie de sanciones mediante una reglamentación que permite el control y facilita el orden (Merton, 1949).

El individuo se vincula como trabajador de la organización, ya que no posee los medios de producción y se busca calcular la conducta y utilizar el mérito como determinante para su acenso en la organización. Así, de la racionalización del trabajo, en la burocracia se derivan no solo virtudes sino, sobre todo, al juzgar de algunos, disfunciones que llegan casi a desplazar sus logros (Merton, 1949), en cuanto generan en el individuo una incapacidad adiestrada, como lo planteara Veblen (1944, citado en Merton, 1949) o una psicosis profesional, como lo planteara Dewey (1938, citado en Merton, 1949). La primera consiste en que las capacidades del individuo se constituyen como inadecuadas en situaciones nuevas, es decir, se convierten en incapacidades; la segunda tiene relación con las preferencias, antipatías y discriminaciones debido a las exigencias que se le imponen en su papel profesional. Esto implica que bajo esta escuela sí hay un lugar para el desarrollo profesional del individuo en la organización, pero dicho desarrollo está supeditado al conocimiento técnico, que puede adquirir mediante la educación, y al cumplimiento estricto de los procedimientos con el fin de acumular méritos que le permitan progresar. El conocimiento técnico se convierte a la vez en medio y obstáculo para su progreso en cuanto le impone limitaciones al individuo, tanto en lo que se refiere a su desarrollo profesional como al desarrollo del trabajo mismo. A través de este se busca su progreso en lo técnico para dejar de lado otro tipo de habilidades y competencias que terminarán por obstaculizar sus funciones y el cumplimiento de los propósitos organizacionales. En consecuencia, podría decirse que en esta escuela el desarrollo profesional del individuo se reduce a lo formal, lo racional y lo objetivo.

\section{La Escuela del Comportamiento}

En esta escuela se reconoce que los procesos de decisión son tan importantes como la acción misma y que el comportamiento de los individuos es intencionado y está dirigido a metas u objetivos que, en últimas, definen lo que hay que hacer. Esto implica seleccionar la meta y el comportamiento que se debe seguir, teniendo en cuenta que este puede ser un fin en sí mismo o un medio para otro fin (Simon, 1988). Para llevar a cabo dichos procesos, será necesario despojar al individuo de gran parte de su autonomía decisoria para sustituirla por un proceso organizativo de toma de decisiones y conseguir la coordinación mediante las líneas de autoridad, la delimitación de las actividades de cada miembro de la organización y la influencia sobre sus comportamientos (Simon, 1988).

Se plantea entonces que la coordinación debe lograrse ya no solo por medio de la cooperación de los individuos, sino mediante la negociación y la influencia sobre sus comportamientos. Aparece aquí un elemento adicional que reconoce que al interior de la organización diferentes divisiones pueden tener diferentes propósitos (Simon, 1988), por lo cual se hace necesaria la negociación de dichos intereses. Si bien se reconoce el papel del individuo como participante clave en la organización, se asume, de manera limitada bajo esta escuela, que estos hacen una contribución a cambio de un salario. Al asumir que los objetivos de la organización son indirectamente objetivos personales de todos sus participantes (Simon, 1988), no se admite que, adicionalmente, al interior de la organización y de cada subdivisión de esta los individuos pueden tener propósitos diferentes, relacionados con su desarrollo y progreso en la organización que también afectan los procesos de decisión. Esto resulta de asumir que el individuo trabaja simplemente a cambio de un salario o que permanece vinculado a la organización simplemente por la deseabilidad percibida de permanecer en la organización o la dificultad percibida de dejarla (March y Simon, 1969).

La toma de decisiones se asume como un proceso racional, pero un elemento interesante en la Escuela del Comportamiento es el reconocimiento de la imposibilidad de alcanzar la racionalidad plena en la toma de decisiones, debido a que los seres humanos no son siempre racionales (Simon, 1988). Dichas limitaciones a la racionalidad objetiva se aceptan e incluyen como parte del proceso de decisiones organizacionales, con el fin de canalizar los comportamientos de los individuos como miembros de un grupo. En este sentido, podría decirse que se considera a los individuos como individuos organizados y, por lo tanto, como un asunto organizacional, pero siempre en su relación con los propósitos organizacionales y como miembros interdependientes en un sistema de relaciones sociales entre los miembros de la organización. En suma, desde esta perspectiva no hay una consideración de individuos con propósitos individuales y decisiones particulares que también deben ser 
negociadas y coordinadas para que confluyan con los propósitos organizacionales.

Para esta escuela, el conocimiento determina las alternativas como primer paso en la toma de decisiones y permite, subsecuentemente, determinar las consecuencias y valorarlas para la toma de decisiones en la organización. Esta escuela también reconoce la existencia de diferentes tipos de racionalidad (Simon, 1988): una decisión es objetivamente racional si es el comportamiento correcto para maximizar unos valores en una situación; al contrario, es subjetivamente racional si maximiza las consecuencias relativas al conocimiento real del sujeto; de otro lado, es conscientemente racional si se hace mediante un proceso consciente de adaptar los medios a los fines; hablamos de una decisión deliberadamente racional si dicho ajuste es deliberado y, por último, una decisión es organizativamente racional si se orienta a los objetivos de la organización o es personalmente racional si se orienta a los del individuo. Bajo este último tipo de racionalidad se encuentra un elemento para considerar los objetivos propios del individuo, dentro los cuales se encontraría su propio desarrollo en la organización. Sin embargo, este elemento no se desarrolla en detalle en la Escuela del Comportamiento, pues presta mayor atención a los demás tipos, especialmente a la racionalidad objetiva, que es limitada, y a la organización como el escenario en el cual "la racionalidad humana consigue sus más elevados objetivos e integraciones del marco institucional en que opera y por el cual es moldeada" (Simon, 1988, p. 97).

Sugiere Simon (1982) que el gerente tiene un papel como tomador de decisiones de la organización, para lo cual requiere práctica, experiencia y aprendizaje con el fin de lograr que la organización, así como cada una de sus partes, enfrenten exitosamente dos tipos de decisiones: programadas, aquellas que son repetitivas y de rutina, y no programadas, es decir, novedosas e inusitadas. En este sentido, cabría decir que el gerente es en sí mismo un individuo que conduce a la organización hacia un propósito común, por ello, la organización se entiende como una coalición compuesta de subcoaliciones en donde sus metas son una serie de restricciones impuestas por un proceso de negociación entre sus miembros y que incluyen la producción, el inventario, las ventas, la participación de mercado y las utilidades (Cyert y March, 1963). Además, como lo plantean Cyert y March (1963), deberá reconocer que los objetivos individuales se hospedan en la mente humana y que será necesario alinearlos con los objetivos de la organización mediante la compra de la conformidad por medio de los pagos efectuados a sus miembros o gracias a la identificación de metas comunes y consensuadas.
Aparecen aquí entonces tres elementos útiles para el análisis del lugar que ocupa el desarrollo del individuo en la organización. En primer lugar, encontramos el gerente como individuo que coordina a otros individuos hacia el cumplimiento de los objetivos de la organización como comunes y consensuados, pero que a la vez tiene sus propios objetivos y deberá conciliarlos con los de la organización. En segundo lugar, tenemos la ausencia de consideración por los procesos de toma de decisiones individuales, en lo referido al progreso de cada miembro individual en la organización, como un elemento para tener en cuenta a la hora de alinear a los individuos con un propósito común. Por último, en la Escuela del Comportamiento se ve a la práctica, la experiencia y al aprendizaje como requisitos para llegar a ser el gerente y el tomador de la decisión, esto es, como medios para el desarrollo profesional del individuo en la organización.

Con respecto al equilibrio en la organización, plantean March y Simon (1969) que este depende en gran medida de los individuos que la conforman por medio de sus relaciones sociales, así como el intercambio de contribuciones por compensaciones que determinan la supervivencia de la organización. No obstante, plantean también que es necesario reconocer que los empleados actúan de una manera subjetiva racional (March y Simon, 1969) y que, por lo tanto, son una fuente de conflicto en cuanto el conflicto individual aparece en las decisiones propias que a su vez afectan la organización (Cyert y March, 1963). Esto implica un reconocimiento de las consecuencias de los temas individuales, pero siempre en relación con los propósitos de la organización en lo referido al conflicto intraindividual, que está estrechamente relacionado con el conflicto individual y tiene como fuentes la inaceptabilidad, la no comparabilidad y la incertidumbre. Dicho de otro modo, para esta escuela es necesario solucionar el problema y persuadir a los individuos sobre objetivos compartidos como procesos analíticos o convenir con ellos y emitir políticas, como procesos de negociación (March y Simon, 1969).

En conclusión, en la Escuela del Comportamiento se reconoce al individuo como poseedor de una intención que se direcciona a unos objetivos, pero se asume de forma algo miope que se trata únicamente de los objetivos de la organización, comunes a todos sus miembros, ignorando la existencia de los objetivos individuales que incluyen el desarrollo profesional en la organización. El reconocimiento de las diferencias individuales es limitado y, más que comprenderlas, esta escuela busca neutralizarlas. Se acepta que la racionalidad en la toma de decisiones en la organización es limitada, pero no se traslada a la toma de decisiones por parte del individuo como actor en dicho proceso, de manera que se ignora a los 
individuos como sujetos y se consideran simplemente actores bajo una perspectiva altamente determinista.

\section{La Escuela de la Contingencia}

La teoría contingente de la organización reconoce la naturaleza sistemática de esta y propone las principales relaciones que deben considerarse para proyectarlas y planearlas, incluyendo la relación entre las diferencias internas y su capacidad para enfrentar las condiciones ambientales (Lawrence y Lorsch, 1973). También considera variables como la estructura y la supervisión como influenciadoras de la estructura organizacional, lo cual contradice los planteamientos de la administración, en cuanto será necesario reconocer que no hay una sola manera de hacer las cosas ni de estructurar las diferentes organizaciones (Woodward, 1975). Asimismo, aparecen los métodos de producción y la tecnología, entre otros, como elementos influenciadores de la estructura de la organización y de las relaciones humanas. Ante esta variedad de factores influyentes en la estructura, se acepta que las "diferentes condiciones externas pueden necesitar características organizativas diferentes y distintos modelos de conducta dentro de una organización eficaz" (Lawrence y Lorsch, 1973, p. 23). Es decir, ya no habrá solamente un one best way, sino diferentes maneras de hacer las cosas, principio denominado all depends.

Dentro de dicha estructura, se reconoce a la organización informal como un intento para alcanzar los propósitos privados y de la organización formal (Burns y Stalker, 1994). Bajo esta escuela, a diferencia de las anteriores, se le da una mayor relevancia a la organización informal y se reconoce que desempeña un papel en la eficiencia de la organización y en el tipo de sistema de gerencia requerido, así como el papel y, de alguna manera, la existencia misma de los propósitos privados en el cumplimiento de los organizacionales. No obstante, el foco continúa estando en la organización informal como un sistema de relaciones sociales de interdependencia y sus efectos sobre la organización, pero no tanto en el proceso de desarrollo del individuo al interior de la organización, dado que sus principales preocupaciones son, como lo plantean Burns y Stalker (1994), la estructura de las organizaciones, su eficiencia y su racionalidad desde una perspectiva mecanicista.

Como lo plantean Burns y Stalker (1994), dicha perspectiva resulta apropiada en condiciones estables que permiten la especialización de funciones, así como las estructuras jerárquicas de control y autoridad, pero ignora la complejidad que impone a la organización la incertidumbre. De esta manera, plantean los autores, en condiciones caracterizadas por ambientes y entornos dinámicos, será más apropiada una perspectiva organicista con el fin de dar respuesta a problemas que cambian continuamente, a partir del conocimiento y la experiencia. Al respecto, dicen Lawrence y Lorsch (1973) que es necesario establecer una conexión entre las condiciones técnicas y económicas externas a la organización con los modelos de organización y administración que conducen al éxito económico, con el fin de identificar el tipo de organización adecuada para esas diversas condiciones externas económicas y de mercado; es decir, buscar las relaciones entre los procesos organizativos y las demandas externas. Podría hacerse aquí una lectura de este planteamiento como un reconocimiento a la necesidad de desarrollar en el individuo ya no solo el conocimiento técnico, como lo planteara la burocracia, sino también la experiencia para lograr que la organización se adapte a dicho entorno dinámico y que el individuo mismo lo haga. No obstante, es importante resaltar que se sigue hablando de los propósitos de la organización y no de aquellos de los individuos al interior de esta.

De igual forma, en la Escuela de la Contingencia, se asume al individuo como participante de la organización, que es un sistema abierto en donde hay relaciones de interdependencia entre la organización formal y la informal (Lawrence y Lorsch, 1973). Se reconoce que la conducta del director está influenciada por diferentes variables como su propia personalidad y su interrelación con la de otros, la naturaleza de la tarea, las relaciones formales, los beneficios, los controles, las ideas que existen en la organización. Adicionalmente, se llama la atención sobre la forma en la que la teoría tradicional de la organización ignoró el papel de las diferencias en las actitudes y la conducta de los miembros de la organización. Sin embargo, el foco continúa en las relaciones sociales entre los individuos y la relación entre la estructura y el entorno de la organización.

Lawrence y Lorsch (1973) plantean que la teoría contingente se ubica en organizaciones de tipo complejo para reducir la confusión en la teoría de la organización e influenciar el diseño y la organización de estas. En este sentido, según los autores, esta teoría busca reconciliar el enfoque clásico y de relaciones humanas. Para ello, reconoce el intercambio entre la organización y su exterior, así como el papel del ambiente como fuente de incertidumbre. Incluye además los argumentos de la tecnología y del tamaño como influenciadores de la estructura (Child, 1995). Sin embargo, no proporciona un marco teórico suficiente para el estudio del desarrollo profesional del individuo en la organización, porque el reconocimiento a la existencia del individuo se hace siempre desde las relaciones sociales y no desde los procesos individuales. Así, denuncia las perspectivas mecanicistas 
de otras escuelas y las critica en pro de reconocer la complejidad de la organización y sus procesos, pero ignora la complejidad del individuo como parte fundamental de dicha complejidad.

\section{La Escuela de las Nuevas Relaciones Humanas}

Según lo plantea Aryris (1975), la organización es un conjunto intrincado de estrategias humanas diseñadas para alcanzar ciertos fines, que son objetivos, así como para mantenerse internamente y adaptarse a su ambiente externo. Esta escuela de la TO asume que la organización es racional y hace demandas racionales a sus empleados, lo cual se plasma en la estructura formal. Además, considera que en la organización existe un sistema de comportamientos derivados de la organización informal, las actividades informales, las necesidades individuales y la combinación de los tres anteriores. Para Montaño Hirose (1991), la Escuela de las Nuevas Relaciones Humanas no es simplemente una extensión de la Escuela de las Relaciones Humanas, ya que, si bien está conformada en torno a la psicología, incorpora planteamientos de escuelas posteriores y acude a planteamientos provenientes de otras disciplinas. En este sentido, esta escuela dará un papel importante a la estructura informal como el resultado de la incapacidad de la organización para facilitar el crecimiento psicológico del individuo, y plantea que la participación se consigue mediante la motivación, pasando de la relación satisfacción/productividad a la motivación/productividad (Montaño Hirose, 1991).

Bajo esta escuela se plantea también una relación de interdependencia entre los superiores y los subordinados, a diferencia de otras escuelas que solo reconocían la dependencia hacia arriba, pero no la interdependencia, y que se constituían como especulaciones teóricas en lugar de investigaciones empíricas sobre los supuestos que gobiernan la conducta humana (McGregor, 1972). Dicha interdependencia, que caracteriza a la sociedad moderna, determinará el equilibrio consistente en "tolerar ciertas formas de dependencia sin molestias excesivas y, al mismo tiempo, mantenerse por su propio pie en algunos aspectos sin demasiada ansiedad" (McGregor, 1972, p. 37). McGregor (1972) propone que la influencia debe darse por medio de métodos adecuados y no solamente desde la autoridad, por lo mismo, destaca a la persuasión e incluso a la ayuda profesional como medios para modificar la capacidad de los individuos con el fin de lograr sus objetivos. Adicionalmente, surgen diversas teorías que buscan explicar el comportamiento del individuo en la organización a partir de sus necesidades. De acuerdo con los aportes de Herzberg (1966), Maslow (1970) y McGregor (1972), los individuos poseen diferentes necesidades y para lograr su cooperación con los objetivos de la organización será necesario obtener en el individuo la satisfacción de sus necesidades y la satisfacción en el trabajo.

McGregor (1972) sugiere que el punto de vista tradicional sobre la dirección y el control pueden denominarse como Teoría X, bajo la cual se concibe el ser humano a partir de su repugnancia intrínseca por el trabajo, por lo cual es necesario obligarlo, controlarlo, dirigirlo y amenazarlo para conseguir su esfuerzo en pro de los objetivos de la organización. Añade también que el individuo carece de ambición y lo define como un ser constantemente insatisfecho, pues entre los diferentes tipos de necesidades que posee siempre habrá alguna, o algunas, sin satisfacer. Con la Teoría X aparece un elemento interesante de análisis y es el reconocimiento de necesidades que tienen que ver con el desarrollo del individuo en diferentes niveles. En este sentido, la teoría menciona las necesidades orgánicas, como el nivel inferior donde se encuentra la necesidad de alimento; las de nivel superior, que realmente pueden usarse para motivarlo e incluyen a las necesidades materiales como la seguridad y la protección contra el peligro, la amenaza y la privación, en este nivel se encuentran también las necesidades sociales que comprenden el sentirse miembro de la organización, asociación, estima, entre otras. Por encima de estas se encuentran las necesidades del yo que son las más importantes para el administrador y para el individuo, y que pueden clasificarse en dos categorías. La primera se refiere a conceptos personales como el respeto y la autoconfianza, la autonomía, la competencia y el saber; la segunda, al prestigio como el reconocimiento, el buen nombre y el respeto por parte de otros. En este nivel de necesidades se puede observar cómo se reconocen en el individuo necesidades de realización personal y se constituyen como un punto de partida interesante para estudiar el desarrollo del individuo. Si bien en la Escuela de las Nuevas Relaciones Humanas se asume al individuo en relación con los demás miembros de la organización y en relación con los objetivos de la organización como propósito común, estos elementos incipientes de las diferencias y necesidades individuales podrían utilizarse para el estudio del individuo en la organización y su desarrollo profesional.

En la Teoría Y propuesta también por McGregor (1972) en contraste a la Teoría X, se sugiere, por primera vez de manera explícita en la TO, la integración de los objetivos individuales con los objetivos de la organización y que, si bien se ha comprendido la importancia del elemento humano, aún no ha logrado incorporarse en el análisis de las organizaciones. Propone además que al ser humano no le disgusta trabajar y que el trabajo puede 
ser una fuente de satisfacción en sí mismo, para lo cual el castigo y el control externo no serán los únicos medios de influencia, ya que el compromiso con los objetivos de la organización se logra mediante las compensaciones asociadas a sus necesidades individuales. De esta forma, surge una posición dinámica con respecto al desarrollo y al crecimiento del ser humano, un recurso con grandes posibilidades que tiene como principio fundamental la integración y el autocontrol, ya que permiten modificar las necesidades y aspiraciones de los miembros en pro de los objetivos de la organización.

Maslow (1970) propuso una teoría positiva de la motivación con una orientación funcionalista que sigue los planteamientos de "James Dewey y se funde con el holismo de Wertheimer, Goldstein y la psicología de la Gestalt, y con el dina- mismo de Freud, Horney, Reich, Jung y Adler" (Maslow, 1970, p. 21). Su teoría es holística y dinámica, y propone la división de las necesidades en una nueva jerarquía que incluye necesidades básicas de seguridad, de amor y pertenencia, de estima, de autorrealización y estéticas. Bajo el nivel de autorrealización aparece la búsqueda del individuo por su realización y se reconocen las necesidades individuales en su máxima expresión (Maslow, 1970). De igual forma, es importante resaltar que la propuesta de este autor presupone la existencia de ciertas condiciones como la libertad de expresión, la justicia, la honestidad, entre otras, como prerrequisito para la satisfacción de las necesidades básicas y el reconocimiento de las diferencias individuales con respecto al lugar que ocupa cada nivel de necesidades como influenciador de su comportamiento, que también ser verá influenciado por estímulos externos.

La teoría de la motivación y la higiene propuesta por Herzberg (1966) toma en consideración a la dualidad de la mente humana al plantear una nueva clasificación de necesidades con el fin de determinar las fuentes de satisfacción e insatisfacción en el trabajo, resaltando que la insatisfacción no es necesariamente la ausencia de satisfacción. Entre los determinantes de la satisfacción, el autor incluye el logro, el reconocimiento, el trabajo, la responsabilidad y el progreso; estos tres últimos serían los más importantes para generar cambios de actitudes duraderos. De esta manera, la propuesta de Herzberg (1966) ofrece un elemento de análisis interesante que podría ser utilizando para el estudio de los procesos de desarrollo profesional del individuo en la organización. No obstante, es importante aclarar que en este, como en los demás autores de esta escuela, prevalece el funcionalismo, pues se piensa al individuo y a su satisfacción en función, no de su propio desarrollo dentro de la organización, sino de su contribución a los objetivos de esta. En este sentido, podría decirse que, de acuerdo con los planteamientos de Argyris (1975), las explicaciones proporcionadas por esta escuela son dinámicas, en cuanto se ocupan de cómo se comporta el individuo en el presente, y no genéticas, ya que no se enfocan en cómo el organismo o el individuo, en este caso, llega a ser lo que es o lo que será. Entonces, como lo dice Blakke (1953, citado en Argyris, 1975), sería importante reconocer que un individuo maduro es aquel predispuesto no solo hacia los fines correctos, que no son más que los propósitos de la organización, sino también hacia el crecimiento propio.

En síntesis, la Escuela de las Nuevas Relaciones Humanas ofrece elementos interesantes desde la motivación y las necesidades del individuo para el análisis del proceso de desarrollo profesional de este en la organización. Sin embargo, es importante señalar que continúa con la racionalidad instrumental y el funcionalismo de otras escuelas, en cuanto reconoce la subjetividad del individuo solo en función del propósito de la organización. De esta manera, será necesario ayudarlo a satisfacer sus necesidades, que van más allá de la compensación, a través del salario para conseguir su cooperación, haciendo cada vez más evidente la concepción de lo humano como recurso y del individuo como activo.

\section{Discusión}

De este análisis conceptual sobre diferentes escuelas de la TO es importante resaltar que "la teoría organizacional no es una sola teoría, sino muchas" (Ibarra-Colado, 2000, p. 277), conformada por una serie de esfuerzos por explicar y comprender a las organizaciones. Las diferentes escuelas aquí presentadas, así como los fundamentos generales de la TO, reconocen la existencia del individuo en la organización, su calidad de participante, su rol como ser social protagonista de las relaciones que ocurren en la organización, los efectos que asume como receptor de los resultados de la organización y su contribución al cumplimiento del propósito de esta. Todas las escuelas, en mayor o menor medida, le otorgan un lugar al individuo, y cada vez que surge una nueva trae consigo nuevos elementos de análisis que resultan interesantes para estudiar y comprender su desarrollo profesional en la organización.

A pesar de sus claras diferencias, se encuentran algunas similitudes acerca del individuo en las organizaciones en las perspectivas de las escuelas mencionadas, las cuales vale la pena señalar. En primer lugar, se habla siempre del propósito de la organización como común a todos sus miembros. De esta forma, se pasa por la dominación, el control, la alineación y la integración, entre otros términos, para referirse a lo que en realidad se constituye como la alienación del individuo en pro del propósito de 
la organización. En segundo lugar, las posibilidades de desarrollo del individuo en la organización se encuentran supeditadas a que este doblegue los intereses propios a los de la organización. Así, se habla del conocimiento técnico en la TO de la Burocracia y de la experiencia en la TO del Comportamiento como los medios para progresar en la organización bajo supuestos parcializados e incompletos que resultan a la vez en disfunciones y obstáculos para el individuo mismo. Esto resulta un elemento común a las teorías de la organización que se caracterizan por su racionalidad instrumental al considerar al individuo en relación con, en función de, en lugar de considerarlo como sujeto y analizar su subjetividad.

$\mathrm{Si}$ bien existen en las diferentes escuelas algunos elementos que podrían resultar útiles y adecuados para estudiar el desarrollo profesional del individuo en la organización, es necesario señalar también que predomina la sobrerracionalización de las organizaciones, el énfasis en los aspectos estáticos y la visión del individuo como carente de ambiciones (Zey-Ferrell, 1981). Dichas ambiciones se hacen presentes solamente en la Escuela de las Nuevas Relaciones Humanas que reconoce la realización como uno de los niveles de necesidades que deben satisfacerse, con el fin de conseguir la satisfacción del individuo en el trabajo y su contribución con los objetivos de la organización.

Como lo plantean Contreras Manrique y De la Rosa Alburqueque (2013), un asunto es y da sentido a lo organizacional si se refiere o es referido por la organización y/o lo organizado. El desarrollo profesional del individuo se presenta en la organización mediante la disposición de los individuos como grupo y de su comportamiento en pro de su realización y desarrollo personal, lo cual ocurre a la vez que persigue los objetivos de la organización. Esto implica que los objetivos personales pasan a ocupar un segundo lugar con relación a los de la organización. Estos objetivos personales solo serán posibles en la medida en que respondan a los objetivos de la organización y se reconozca el lugar que ocupa el desarrollo individual en dicha organización y sus efectos sobre el desarrollo de la misma.

\section{Conclusión}

Las perspectivas dominantes sobre la organización se enfocan en su eficiencia, las metas, el consenso, lo estático, entre otros, y dejan de lado otros aspectos importantes como el cambio, el poder, los conflictos de intereses y la ambición individual. En consecuencia, y para estudiar el desarrollo profesional del individuo en la organización, sería necesario dejar de lado el racionalismo funcional encontrado en las teorías aquí mencionadas.
Para ello, el paradigma radical humanista, que es completamente opuesto al funcionalismo, podría contribuir a entender al individuo desde una perspectiva subjetivista. Adicionalmente, sería útil abandonar el método cuantitativo y el positivismo de la TO, para asumir el estudio del individuo como nivel de análisis dentro de los asuntos organizacionales, e incluso adoptar otros métodos predominantemente cualitativos, como el interpretativismo, con el fin de comprender los procesos de desarrollo profesional del individuo en la organización. Esto implicará, además, aceptar este fenómeno desde una perspectiva epistemológicamente constructivista, bajo la cual se admita que los individuos son quienes crean a la sociedad y a las organizaciones.

\section{Referencias}

Argyris, C. (1975). The impact of the Formal Organization upon the individual. En Organization theory (pp. 261-278). Penguin Books.

Barba Álvarez, A. (2010). Frederick Winslow Taylor y la administración científica: contexto, realidad y mitos. Gestión y Estrategia, 38, 17-29.

Barnard, C. I. (1959). Las funciones de los elementos dirigentes. Documentación Administrativa. https://doi. org/10.24965/da.vi23.1296

Burns, T., \& Stalker, G. M. (1994). The management of innovation. Oxford University Press.

Child, J. (1995). Organizational structure, environment and performance: the role of strategic choice. En Contingency theory (pp. 399-420). Cambridge.

Contreras Manrique, J. C., \& De la Rosa Alburqueque, A. (2013). Organizaciones y políticas públicas. Elementos para trabajar una perspectiva organizacional de las políticas públicas. En Hacia la perspectiva organizacional de la política pública. Recortes y orientaciones iniciales (pp. 13-56). Fontamara.

Coriat, B. (2000). El taller y el cronómetro. Ensayo sobre el taylorismo, el fordismo y la producción en masa. Siglo XXI.

Cyert, R. M., \& March, J. G. (1963). A Behavioral Theory of the Firm. Prentice-Hall, Inc.

Desmarez, P. (1986). La sociología industrial, heredera de la termodinámica del equilibrio. En Teoría de la organización: fundamentos y controversias (pp. 27-38). Universidad Autónoma Metropolitana.

Durand, C. (1979). Los inconvenientes técnicos del Taylorismo. En El trabajo encadenado. Organización del trabajo y dominación social (pp. 59-68). H. Blume Ediciones.

Gonzales-Miranda, D. R., \& Gentilin, M. (2012). Panorama del estudio de las organizaciones. Una caracterización del campo organizacional con base en tres ejes temáticos. Revista Análisis Organizacional, 1(5), 1-28. 
Hall, R. (1996a). Los resultados de las organizaciones. En Organizaciones. Estructuras, procesos y resultados (pp. 1-28). Prentice-Hall.

Hall, R. (1996b). Sobre la naturaleza y tipos de organizaciones. En Organizaciones. Estructuras, procesos y resultados (pp. 29-9). Prentice-Hall.

Herzberg, F. (1966). Work and the nature of man. Thomas Y. Crowell Company.

Ibarra-Colado, E. (2000). Teoría de la organización, mapa conceptual de un territorio en disputa. En Tratado Latinoamericano de Sociología del Trabajo (pp. 245284). COLMEX, FLACSO, UAM Y FCE.

Ibarra-Colado, E., \& Montaño, L. (1991). Organización y administración. Una lectura crítica para América Latina. En Ensayos críticos para el estudio de las organizaciones en México (pp. 7-25). Miguel Ángel Porrúa: UAM-I.

Lawrence, P. R., \& Lorsch. (1973). Organización y ambiente. Editorial Labor S. A.

March, J. G., \& Simon, H. A. (1969). Teoría de la Organización. Ediciones Ariel.

Maslow, A. H. (1970). Motivation and personality. Harper \& Row Publishers.

Mayo, E. (1972). Problemas humanos de una civilización industrial. Nueva Visión.

Mayo, E. (1977). Problemas sociales de una civilización industrial. Nueva Visión.

McGregor, D. (1972). El aspecto humano de las empresas. Editorial Diana.

Merton, R. K. (1949). Estructura burocrática y personalidad. En Teoría y estructuras sociales (pp. 275-286). Fondo de Cultura Económica.

Montaño Hirose, L. (1991). Las Nuevas Relaciones Humanas: un falso reto a la democracia. En Ensayos criticos para el estudio de las organizaciones (pp. 69-102). Miguel Ángel Porrúa: UAM-I
Mouzelis, N. P. (1967). El tipo ideal de burocracia. En Organizacion y burocracia (pp. 45-61). Ediciones Península.

Porter, L. W., Lawler, E. E., \& Hackman, J. R. (1975). Behavior in organizations. McGraw-Hill.

Perrow, C. (1980). La historia del Zoológico o La vida en el arenal organizativo. En Control e ideología en las organizaciones (pp. 293-314). Fondo de Cultura Económica.

Roethlisberger, F. J., \& Dickson, W. J. (1966). Management and the worker. Harvard University Press.

Scott, R. W. (1981). The subject is organizations. En Organizations, rational, natural and open systems (pp. 3-26). Prentice-Hall.

Simon, H. A. (1982). Los procesos de la decisión gerencial. En La nueva ciencia de la dirección gerencial (pp. 36-45). El Ateneo.

Simon, H. A. (1988). El comportamiento administrativo. Estudio de los procesos decisorios en la organización administrativa. Aguilar.

Taylor, F. (1997). ¿Qué es la administración científica? y Principios de administración científica. En Clásicos en administración (pp. 77-107). Limusa.

Weber, M. (1922). Sociología de la Dominación. En Economía y Sociedad (pp. 695-752). Fondo de Cultura Económica.

Woodward, J. (1975). Management and technology. En Organization theory (pp. 56-71). Penguin Books.

Zey-Ferrell, M. (1981). Criticisms of the dominant Perspective on Organizations. The Sociological Quarterly, 22, 181-205. 